\title{
Association between alcohol dehydrogenase-2 gene polymorphism and esophageal cancer risk: a meta-analysis
}

\author{
Ning Mao', Siyao Nie², Bin Hong ${ }^{1}$, Chao Li ${ }^{1}$, Xueyuan Shen ${ }^{1}$ and Tao Xiong ${ }^{1 *}$
}

\begin{abstract}
Background: It has been shown that gene polymorphisms may play an important role in the carcinogenesis of esophageal cancer. This study is to investigate the role of alcohol dehydrogenase 1B (ADH1B) gene Arg47His polymorphism in esophageal cancer susceptibility.

Methods: Case-control studies published between January 2000 and June 2015 were searched to retrieve relevant articles. The pooled odds ratio (OR) and $95 \%$ confidence interval (Cl) were employed to calculate the strength of association.

Results: A total of 23 relevant articles were finally selected for the analysis, including 9338 esophageal cancer patients and 14,896 matched controls. Overall, we found that the $47 \mathrm{His}$ allele was significant associated with the decreased risk of esophageal cancer when compared with the 47Arg allele in total populations (A vs. G: OR=0. $67,95 \% \mathrm{Cl}=0.59-0.76, P<0.00001)$. This protective relationship was observed under other genetic models as well $(P<0.00001)$. Subgroup analysis by ethnicity showed that ADH1B Arg47His variant was associated with the decreased esophageal cancer risk under all the genetic models $(P<0.00001)$ among Asians, especially in Chinese and Japanese; while in non-Asians, no significant correlation was detected in any genetic models $(P>0.05)$. Furthermore, $\operatorname{Arg} / \operatorname{Arg}$ genotype of ADH1B Arg47His variant combined with drinking, smoking and males appeared to show a high risk in patients with esophageal cancer.
\end{abstract}

Conclusions: Our results suggested that ADH1B gene Arg47His variant was associated with the decreased esophageal cancer risk. Genetic-environmental interaction should be further considered in the future researches.

Keywords: Esophageal cancer, Alcohol dehydrogenase 1B, Polymorphism, Meta-analysis

\section{Background}

Esophageal cancer (or oesophageal cancer), a type of cancer arising from the esophagus, is the eighth most common cancer and the sixth leading cause of cancerrelated death worldwide [1]. Squamous cell carcinomas (SCC), which is more common in the developing world, and adenocarcinomas (AC), which is more common in the developed world, are two main forms of histologically confirmed esophageal cancer [2, 3]. The clinical symptoms include difficulty in swallowing, enlarged lymph glands around the collarbone, a dry cough, weight

\footnotetext{
* Correspondence: xisontao@yeah.net

${ }^{1}$ Department of Cardiothoracic Surgery, Yongchuan Hospital of Chongqing Medical University, No. 439 Xuanhua Road, Yongchuan District, Chongqing 402160, China

Full list of author information is available at the end of the article
}

loss, and possibly hematemesis [4]. The established risk factors for this disease are environmental factors (alcohol drinking, smoking, infecting bacteria or virus), genetic factors (mutations in enzymes that metabolize alcohol), cultural factors (high-temperature food items such as pork braised in brown sauce and old stocked rice), obesity and gastroesophageal reflux [5-8]. The incidence of esophageal cancer is threefold higher in men than women [9] and is high in east Asia, southern Africa, and southern Europe while is low in North America and other parts of Europe [10]. The highest reported incidence and mortality rates occur in China, which is almost 20 to 30 times higher than that in the USA [11]. According to cancer statistics, there will be an estimated 16,980 new cases and 15,590 deaths in both 
sexes in the USA in 2015 [12]. Although many advances in diagnosis and treatment such as endoscopic resection, chemotherapy, and surgery have the potential to substantially reduce mortality and morbidity [13, 14], the prognosis of patients with esophageal cancer remains poor and the 5-year survival rate is still low, ranging from 15 to $25 \%$ [15, 16]. Therefore, identifying new biomarkers for early diagnosis and treatment is vital to decide therapeutic options, improve treatment efficiency, and predict prognosis [17].

Evidences have shown that gene polymorphisms may play an important role in the carcinogenesis of esophageal cancer [18]. Alcohol dehydrogenase gene (ADH), at chromosome 4, is a key cytosolic enzyme for ethanol [19]. It encodes at least seven ADH isoforms (ADH1ADH7), each with slightly different properties [20], and may be involved in the metabolic pathways of several neurotransmitters [21]. The ADH1B (also known as $\mathrm{ADH} 2)$ gene is located on human chromosome 4q21q23. It is the locus responsible for most of the ADH activity on ethanol in the liver [22]. Single nucleotide polymorphisms (SNPs) occurring in this gene may be capable of altering ethanol metabolism, and individuals expressing the ADH1B variants would have different alcohol elimination rates [23]. One of the most studied SNP was Arg47His (rs1229984), a G to A base transition in exon 3 leading to the substitution of arginine (ADH1B*1) to histidine (ADH1B*2) at codon 47th position. ADH1B*2 is common in more than $90 \%$ of Asians and reduced their risk for alcoholism but fewer than $20 \%$ of Caucasians or Africans [24]. This variant was shown to be strongly associated with alcohol dependence, abuse, consumption, and alcohol-induced liver diseases $[25,26]$.

Several studies have identified the relationship between ADH1B polymorphism and esophageal cancer susceptibility, but the consistent results were not obtained. For example, Ito et al. demonstrated that ADH1B Arg47His variant was associated with esophageal cancer in Japanese and might be used in personalized prevention programs [27], while Ma et al. did not show significant associations between variations in the $\mathrm{ADH} 1 \mathrm{~B}$ gene and esophageal squamous cell carcinoma (ESCC) risk in Chinese [28]. Although previous meta-analyses were performed to evaluate this association [29-31], limited articles were selected, only Asian population was used for analysis, and some repeated participants were included. Furthermore, Asians and Caucasians may have different incidence, distribution, and susceptibilities to esophageal cancer due to different heritage backgrounds [32]. All these factors may influence the reliable of the results. Therefore, we conducted the present meta-analysis to review all the published articles among any ethnicities to obtain a relative reliable result.

\section{Methods}

\section{Identification of relevant studies}

Electronic databases of PubMed, Web of Science, Medline, Embase, CNKI (China National Knowledge Internet), and Wanfang were comprehensively searched to retrieve relevant articles published between January 2000 and June 2015. The MeSH terms were as follows: "esophageal cancer or oesophageal cancer or esophageal squamous cell carcinomas or esophageal adenocarcinomas," "alcohol dehydrogenase or ADH1B or ADH2," and "polymorphism or variant or mutation" as well as their combinations. The equivalent Chinese characters were used in Chinese database. We also manually searched the references of included studies in order to obtain more related articles. Our analysis only considered studies that were written in English and Chinese.

\section{Inclusion and exclusion criteria}

The eligible articles must meet the following criteria. The inclusion criteria were as follows: (1) case-control studies evaluating the correlation of ADH1B Arg47His polymorphism in esophageal cancer occurrence; (2) patents with esophageal cancer should be diagnosed clinically and confirmed histologically, the controls should be age-, ethnicity-matched participants without malignancy and digestive and chronic diseases; (3) the genotype information were available to extract; and (4) the genotypes of controls were consistent with Hardy-Weinberg equilibrium (HWE). The exclusion criteria were as follows: (1) duplicate articles from the same authors or laboratories or conducted among the same populations; (2) conference reports or reviews; (3) without control group; and (4) data could not be extracted.

\section{Data extraction}

Two of our authors independently assessed the quality of selected studies. Each item of a single study should be achieved a final consensus. The extracted information was as follow: the name of first author, published year, country, ethnicity, mean age, sample size, genotype method, alleles and genotypes distribution, source of cases and controls, and HWE of genotypes in controls.

\section{Statistical analysis}

The pooled odds ratio (OR) with its $95 \%$ confidence interval $(\mathrm{CI})$ were employed to estimate the association between ADH1B Arg47His variant and esophageal carcinoma susceptibility. The $Z$ test was used to determine the significance of ORs, and $P$ value was less than 0.05 considered as statistically significance. The allelic model (His vs. Arg), the homologous model (His/His vs. Arg/ Arg), the heterogeneous model (Arg/His vs. Arg/Arg), the dominant model (His/His+Arg/His vs. Arg/Arg) and the recessive model (His/His vs. Arg/His+Arg/Arg) were 
examined. The $Q$ test and the $I^{2}$ test were used to assess the degree and proportion of between-study heterogeneity, respectively. The fixed-effect model was used when the $P$ value of the $Q$ test was more than 0.10 and the $I^{2}$ of the $I^{2}$ test was less than $50 \%$; otherwise, the random-effect model was used. All statistical analyses were conducted in Review Manager 5.2 (the Cochrane Collaboration, Oxford, England). All the tests were two-sided.

\section{Results}

\section{Characteristics of included studies}

We firstly identified 105 articles. After the inclusion and exclusion criteria filtering, a total of 23 relevant articles were finally screened out, including 9338 esophageal cancer patients and 14,896 matched controls. Figure 1 presented the selection process. Of the 23 articles, two were written in Chinese [33, 34] and 21 were in English [35-55]. The participants of Hashibe et al.'s study were from five countries (Romania, Poland, Russia, Slovakia, and Czech Republic) [38], while other studies were from seven countries (Japan, Thailand, Iran, Kashmir, Sudan, Dutch and China), respectively. There were two sources of controls: population-based controls and hospitalbased controls. The ADH1B Arg47His variant was measured in eight methods. Table 1 listed the main characteristics of included studies. Table 2 displayed the alleles and genotypes information of ADH1B Arg47His variant in each included studies.
Overall association between ADH1B Arg47His polymorphism and esophageal cancer risk

A significant heterogeneity among all the included studies was observed $\left(P<0.01\right.$ and $\left.I^{2}>50 \%\right)$, and the random-effect model was used. Table 3 listed the results of the association between the ADH1B Arg47His polymorphism and esophageal cancer susceptibility. Overall, we found that the frequency of $47 \mathrm{His}$ allele was higher than the 47Arg allele in both cases and controls, and the statistical analysis demonstrated that the $47 \mathrm{His}$ allele was significant associated with the decreased risk of esophageal cancer in total populations (His vs. Arg: $\mathrm{OR}=0.67$, $95 \% \mathrm{CI}=0.59-0.76, P<0.00001)$ as shown in Fig. 2 . This significant protective relationship was also identified under other genetic models $(P<0.00001)$. Subgroup analysis by ethnicity showed that this polymorphism revealed an ethnic difference and geographic variance. In Asians, ADH1B 47His variant was shown to be associated with the decreased esophageal cancer risk under all the genetic models $(P<0.00001)$; while in non-Asians, no significant correlation was detected in any genetic models $(P>0.05)$. Figure 3 showed the result of the His carriers of the His/ His and Arg/His genotypes compared with the Arg/Arg genotype in both groups. Furthermore, we also considered the role of this genetic variant in esophageal cancer among different countries. There were 12 articles in China (5485 patients and 6982 controls), five in Japan (2319 patients and 5213 controls), and six in other countries. Our result demonstrated that $\mathrm{ADH1B}$ Arg47His variant was a protective factor for cancer risk in Chinese and Japanese populations as shown in Fig. 4.

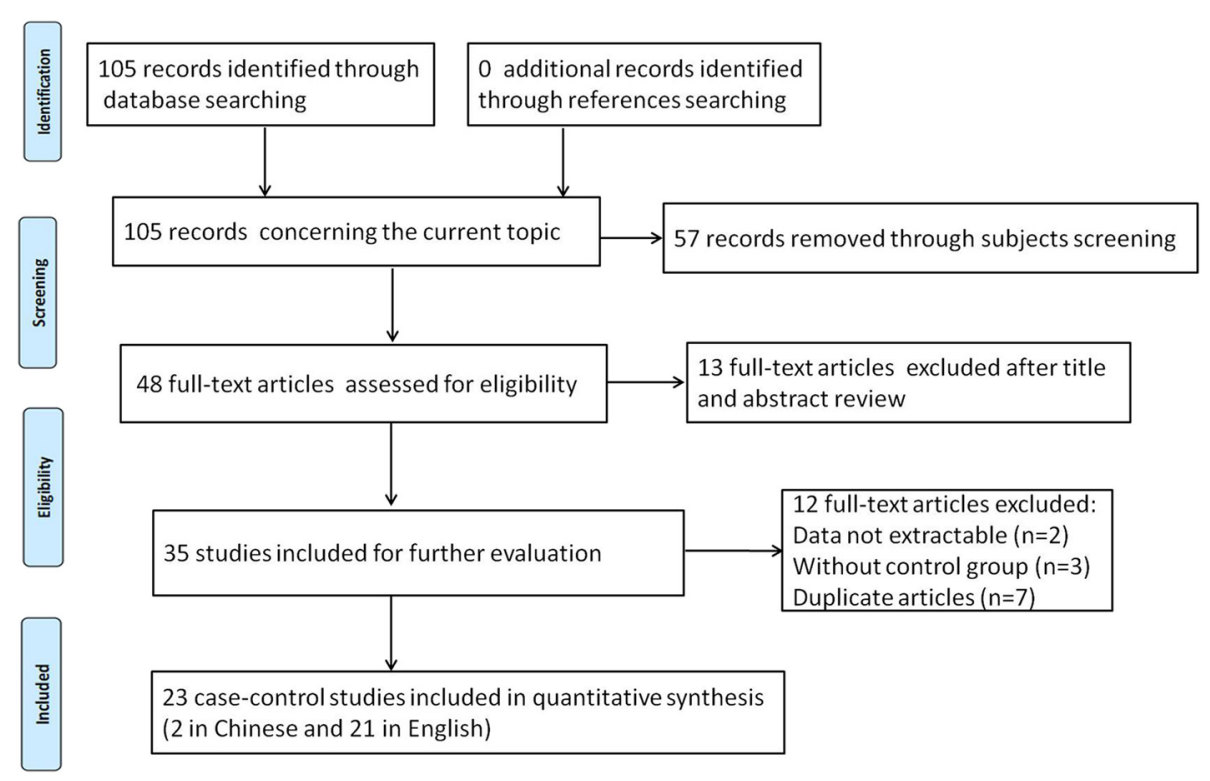

Fig. 1 Flow diagram of the selection process 
Table 1 Main characteristics of included studies in this meta-analysis

\begin{tabular}{|c|c|c|c|c|c|c|c|c|c|c|}
\hline \multirow[t]{2}{*}{ First author } & \multirow[t]{2}{*}{ Year } & \multirow[t]{2}{*}{ Country } & \multirow[t]{2}{*}{ Ethnicity } & \multicolumn{2}{|l|}{ Mean age } & \multicolumn{2}{|c|}{ Sample size } & \multicolumn{2}{|l|}{ Source of } & \multirow{2}{*}{$\begin{array}{l}\text { Genotyping } \\
\text { methods }\end{array}$} \\
\hline & & & & Cases & Controls & Cases & Controls & Cases & Controls & \\
\hline Yokoyama A & 2001 & Japan & Asian & $56 \pm 7$ & $53 \pm 8$ & 112 & 526 & ESCC & $\mathrm{HB}$ & PCR-RFLP \\
\hline Boonyaphiphat P & 2002 & Thailand & Asian & $64.0 \pm 9.7$ & $64.5 \pm 12.3$ & 202 & 261 & ESCC & $\mathrm{HB}$ & APLP \\
\hline Chao YC & 2003 & China (Taiwan) & Asian & $64.6 \pm 12.3$ & $53.0 \pm 18.8$ & 88 & 105 & EC & $\mathrm{HB}$ & PCR-RFLP \\
\hline Yokoyama T & 2003 & Japan & Asian & $61.7 \pm 7.9$ & $58.9 \pm 7.1$ & 233 & 610 & ESCC & PB & PCR-RFLP \\
\hline Chen HG & 2005 & China & Asian & $58.06(35-80)$ & $58.53(35-82)$ & 137 & 137 & EC & PB & PCR \\
\hline Yang CX & 2005 & Japan & Asian & $61.4 \pm 0.6$ & $61.4 \pm 0.4$ & 165 & 495 & ESCC/EAC & $H B$ & PCR-CTPP \\
\hline Hashibe M & 2006 & Mixed & Caucasian & $56.7 \pm 2.1$ & $58.9 \pm 3.4$ & 167 & 887 & ESCC & PB & TaqMan \\
\hline Yang SJ & 2007 & China & Asian & $58.3 \pm 8.3$ & $52.8 \pm 13.2$ & 191 & 198 & EC & PB & PCR-CTPP \\
\hline Guo YM & 2008 & China & Asian & $60.2 \pm 8.9$ & $59.7 \pm 9.7$ & 80 & 480 & ESCC & PB & PCR-RFLP \\
\hline Lee $\mathrm{CH}$ & 2008 & China (Taiwan) & Asian & $60.2 \pm 3.8$ & $61.3 \pm 3.9$ & 406 & 656 & ESCC & $\mathrm{HB}$ & PCR-RFLP \\
\hline Akbai & 2009 & Iran & Asian & $63.6(25-89)$ & $55.2(24-90)$ & 746 & 1373 & ESCC & PB & Sequenom \\
\hline Cui $\mathrm{R}$ & 2009 & Japan & Asian & $64.2 \pm 8.7$ & $59.7 \pm 16.2$ & 1070 & 2836 & ESCC & PB & BeadChip \\
\hline Ding JH & 2010 & China & Asian & $68.2 \pm 5.6$ & $69.8 \pm 7.3$ & 221 & 191 & EC & PB & PCR-DHPLC \\
\hline Tanaka F & 2010 & Japan & Asian & $67.2 \pm 7.3$ & $66.5 \pm 7.2$ & 742 & 820 & OscC & PB & BRLMM \\
\hline lqbal B & 2011 & Kashmir & Asian & NA & NA & 50 & 50 & EC & PB & Sequencing \\
\hline Wang YL & 2011 & China & Asian & $57.2 \pm 9.4$ & $56.1 \pm 7.3$ & 81 & 162 & EC & PB & PCR-CTPP \\
\hline Gu HY & 2012 & China & Asian & $62.5 \pm 6.2$ & $63.8 \pm 7.1$ & 380 & 380 & ESCC & $\mathrm{HB}$ & MALDI-ToF-MS \\
\hline Dura P & 2013 & Dutch & Caucasian & $65.0 \pm 10.9$ & $64.9 \pm 11.1$ & 351 & 430 & ESCC/EAC & PB & TaqMan \\
\hline Gao Y & 2013 & China & Asian & NA & NA & 2139 & 2273 & ESCC & PB & TaqMan \\
\hline Wu M & 2013 & China & Asian & $63.7 \pm 9.4$ & $63.7 \pm 10.3$ & 846 & 1079 & EC & PB & Sequencing \\
\hline Hou AM & 2014 & China & Asian & $56.5 \pm 5.5$ & $58.7 \pm 6.3$ & 110 & 110 & ESCC/EAC & PB & PCR-RFLP \\
\hline Ye B & 2014 & China & Asian & $58.7 \pm 6.4$ & $59.9 \pm 7.2$ & 1001 & 1391 & ESCC & $\mathrm{HB}$ & PCR-RFLP \\
\hline Babiker H & 2015 & Sudan & Africa & 56.41 & 47.87 & 134 & 233 & ESCC/EAC & PB & PCR-RFLP \\
\hline
\end{tabular}

$N A$ not available, ESCC esophageal squamous cell carcinoma, $E A C$ esophageal adenocarcinoma, OSCC oesophageal squamous cell carcinoma, $H B$ hospital-based, $P B$ population-based, NP normal healthy population, $A P L P$ amplified product length polymorphism method, $P C R$-CTPP polymerase chain reaction with the confronting two-pair primer, $P C R-D H P L C$ polymerase chain reaction and denaturing high-performance liquid chromatography, BRLMM Bayesian robust linear model with Mahalanobis algorithm, MALDI-TOF-MS matrix-assisted laser desorption/ionization-time-of-flight mass spectrometry

Combined effect of ADH1B Arg47His variant and alcohol drinking, tobacco smoking, and gender difference on esophageal cancer risk

Fifteen studies estimated the combined effect of ADH1B Arg47His polymorphism and alcohol drinking on esophageal cancer risk. However, the relevant data could be extracted only from 12 studies, including 3545 patients and 6909 controls. Based on these data, we could only assess the combined effect on esophageal cancer susceptibility under the Arg/Arg versus His/His+Arg/His model. We divided the subjects into two groups: non-drinking group (1134 cases and 3526 controls) and drinking group (2411 cases and 3383 controls). Our results found that Arg/Arg genotype compared with the His carrier of His/His and Arg/His genotypes was significantly associated with the increased higher risk of esophageal cancer in the drinking group $(\mathrm{OR}=3.15,95 \% \mathrm{CI}=2.66-$ $3.74, P<0.00001)$ and lower in the non-drinking group $(\mathrm{OR}=1.71,95 \% \mathrm{CI}=1.23-2.38, P=0.001)$ as shown in Fig. 5.

Four articles considered the combined effect of ADH1B Arg47His polymorphism and tobacco smoking on esophageal cancer susceptibility, containing 2382 patients and 4792 controls. Our result demonstrated that the Arg/Arg genotype was associated with esophageal cancer occurrence in both non-smokers $(\mathrm{OR}=2.40$, $95 \% \mathrm{CI}=1.44-3.98, P=0.0007)$ and smokers $(\mathrm{OR}=$ $3.35,95 \% \mathrm{CI}=2.22-5.05, P<0.00001)$ in the randomeffect model as shown in Fig. 6, and this relationship was a bit stronger in smokers than that in non-smokers.

Six articles were selected for gender variance (five for males and two for females). Our result showed that the Arg/Arg genotype of ADH1B Arg47His variant increased the esophageal cancer risk in male patients $(\mathrm{OR}=3.44,95 \% \mathrm{CI}=2.42-4.89, P<0.0001)$, while not in female patients $(\mathrm{OR}=1.62,95 \% \mathrm{CI}=0.90-2.91, P=$ 0.11 ) as shown in Fig. 7. 
Table 2 Distribution of alleles and genotypes in each included studies

\begin{tabular}{|c|c|c|c|c|c|c|c|c|c|c|c|}
\hline \multirow[t]{3}{*}{ First author } & \multicolumn{5}{|l|}{ Cases } & \multicolumn{6}{|l|}{ Controls } \\
\hline & Arg/Arg & Arg/His & $\mathrm{His} / \mathrm{His}$ & Arg & His & Arg/Arg & $\mathrm{Arg} / \mathrm{His}$ & $\mathrm{His} / \mathrm{His}$ & $\operatorname{Arg}$ & His & HWE \\
\hline & $\left({ }^{*} 1 /{ }^{*} 1\right)$ & $\left({ }^{*} 1 /{ }^{*} 2\right)$ & $\left({ }^{*} 2 /{ }^{*} 2\right)$ & $\left({ }^{*} 1\right)$ & $\left({ }^{*} 2\right)$ & $\left({ }^{*} 1 /{ }^{*} 1\right)$ & $\left({ }^{*} 1 / *\right)$ & $\left({ }^{*} 2 /{ }^{*} 2\right)$ & $\left({ }^{*} 1\right)$ & $\left({ }^{*} 2\right)$ & \\
\hline Yokoyama A & 56 & $56^{*}$ & & - & - & 145 & $381^{*}$ & & & - & - \\
\hline Boonyaphiphat $P$ & 101 & 86 & 15 & 288 & 116 & 94 & 139 & 28 & 327 & 195 & 0.08 \\
\hline Chao YC & 19 & 41 & 28 & 79 & 97 & 7 & 43 & 55 & 57 & 153 & 0.94 \\
\hline Yokoyama T & 51 & 73 & 109 & 175 & 291 & 31 & 212 & 367 & 274 & 946 & 0.99 \\
\hline Chen HG & 26 & 54 & 57 & 106 & 168 & 14 & 49 & 74 & 77 & 197 & 0.40 \\
\hline Yang CX & 6 & 85 & 74 & 97 & 233 & 22 & 168 & 304 & 212 & 776 & 0.98 \\
\hline Hashibe M & 163 & $4^{*}$ & & - & - & 792 & $95^{*}$ & & - & - & - \\
\hline Yang SJ & 33 & 80 & 78 & 146 & 236 & 22 & 76 & 100 & 120 & 276 & 0.44 \\
\hline Guo YM & 17 & 25 & 38 & 59 & 101 & 24 & 168 & 288 & 216 & 744 & 0.99 \\
\hline Lee $\mathrm{CH}$ & 117 & 149 & 140 & 383 & 429 & 46 & 275 & 335 & 367 & 945 & 0.59 \\
\hline Akbai & 21 & 232 & 490 & 274 & 1212 & 73 & 471 & 827 & 617 & 2125 & 0.86 \\
\hline Cui $\mathrm{R}$ & 194 & 363 & 510 & 751 & 1383 & 151 & 986 & 1626 & 1288 & 4238 & 0.99 \\
\hline Ding JH & 19 & 96 & 106 & 134 & 308 & 8 & 75 & 108 & 91 & 291 & 0.53 \\
\hline Tanaka F & 149 & 237 & 356 & 535 & 949 & 49 & 287 & 484 & 385 & 1255 & 0.76 \\
\hline lqbal B & 12 & 36 & 2 & 60 & 40 & 12 & 37 & 1 & 61 & 39 & 0.30 \\
\hline Wang YL & 15 & 34 & 33 & 64 & 100 & 17 & 67 & 78 & 101 & 223 & 0.90 \\
\hline Gu HY & 53 & 168 & 158 & 274 & 484 & 26 & 170 & 182 & 222 & 534 & 0.26 \\
\hline Dura P & 326 & 20 & 0 & 672 & 20 & 406 & 23 & 0 & 835 & 23 & 0.85 \\
\hline Gao Y & 252 & 907 & 939 & 1411 & 2785 & 199 & 909 & 1155 & 1307 & 3219 & 0.57 \\
\hline Wu M & 138 & 309 & 355 & 585 & 1019 & 101 & 410 & 510 & 612 & 1430 & 0.38 \\
\hline Hou AM & $59^{*}$ & & 51 & - & - & $48^{*}$ & & 62 & - & - & - \\
\hline Ye B & 224 & 400 & 377 & 848 & 1154 & 150 & 578 & 663 & 878 & 1904 & 0.36 \\
\hline Babiker H & 115 & 8 & 2 & 238 & 12 & 188 & 18 & 4 & 394 & 26 & 0.06 \\
\hline
\end{tabular}

HWE Hardy-Weinberg Equilibrium;-, not applicable; ${ }^{*}$ for Yokoyama A and Hashibe M means His/His+Arg/His; *for Hou AM means Arg/His+Arg/Arg

\section{Sensitivity analysis and publication bias}

We deleted a single included study at a time to verify whether our results were influenced by each study. The result showed that even though the betweenstudy heterogeneity was reduced, the pooled ORs were not significantly changed, indicating that there was no publication bias in the present meta-analysis. The funnel plot among the total population under the dominant model further reveal no publication bias as shown in Fig. 8.

\section{Discussion}

In this meta-analysis, we totally screened out 23 relevant articles. Our results showed that the ADH1B Arg47His variant was correlated with the decreased occurrence of esophageal cancer under all the genetic models among total population. Subgroup analysis by ethnicity demonstrated that this variant was associated with the decreased cancer risk in Asian populations as well, especially in Chinese and Japanese. However, in non-Asian population, no significant relationship was observed. Furthermore, we found that the combined effect of Arg/Arg genotype in ADH1B Arg47His variant and alcohol drinking, tobacco smoking, and male patients revealed a higher risk on esophageal cancer risk when compared with the His carrier of His/His and Arg/His genotypes, respectively. Our results were consistent with previous meta-analyses which showed that ADH1B*47Arg could significantly increase the risk of ESCC in Asians, especially when coupled with alcohol drinking [29-31].

Esophageal cancer is considered as a serious malignancy worldwide for its extremely aggressive nature and poor survival rate [56]. It is a multistep, multifactorial disease, and the incidence is varied due to the geographical diversities; thus, the therapeutic management might be different as well. Genetic variations might be involved in esophageal cancer development and predict the prognosis [57]. Studies have shown that ADH gene might play a role in carcinogenesis. $\mathrm{ADH}$ constitutes a large family of enzymes and is a metabolic barrier against selfadminister ethanol [58]. ADH isoenzyme is responsible for the reversible oxidation of alcohols to acetaldehyde, 
Table 3 Meta-analysis of the overall association between ADH1B Arg47His polymorphism and esophageal cancer risk

\begin{tabular}{|c|c|c|c|c|c|c|c|}
\hline \multirow[t]{2}{*}{ Classification } & \multirow[t]{2}{*}{ Comparisons } & \multirow[t]{2}{*}{ Number of included studies } & \multicolumn{2}{|c|}{ Test of association } & \multicolumn{3}{|c|}{ Test of heterogeneity } \\
\hline & & & OR (95\% Cl) & $P$ & $\mathrm{Ph}$ & $1^{2}$ & Model \\
\hline \multirow[t]{5}{*}{ Total } & His vs. Arg & 20 & $0.67(0.59,0.76)$ & $<0.00001$ & $<0.00001$ & $87 \%$ & $\mathrm{R}$ \\
\hline & His/His vs. Arg/Arg & 20 & $0.40(0.30,0.54)$ & $<0.00001$ & $<0.00001$ & $86 \%$ & $\mathrm{R}$ \\
\hline & Arg/His vs. Arg/Arg & 20 & $0.52(0.40,0.68)$ & $<0.00001$ & $<0.00001$ & $85 \%$ & $\mathrm{R}$ \\
\hline & $\mathrm{His} / \mathrm{His}+\mathrm{Arg} / \mathrm{His}$ vs. Arg/Arg & 22 & $0.46(0.36,0.59)$ & $<0.00001$ & $<0.00001$ & $86 \%$ & $\mathrm{R}$ \\
\hline & His/His vs. Arg/His+Arg/Arg & 21 & $0.69(0.61,0.77)$ & $<0.00001$ & $<0.00001$ & $69 \%$ & $\mathrm{R}$ \\
\hline \multirow[t]{5}{*}{ Asian } & His vs. Arg & 18 & $0.66(0.57,0.75)$ & $<0.00001$ & $<0.00001$ & $88 \%$ & $\mathrm{R}$ \\
\hline & His/His vs. Arg/Arg & 18 & $0.40(0.30,0.54)$ & $<0.00001$ & $<0.00001$ & $87 \%$ & $\mathrm{R}$ \\
\hline & Arg/His vs. Arg/Arg & 18 & $0.49(0.38,0.65)$ & $<0.00001$ & $<0.00001$ & $86 \%$ & $\mathrm{R}$ \\
\hline & $\mathrm{His} / \mathrm{His}+\mathrm{Arg} / \mathrm{His}$ vs. Arg/Arg & 19 & $0.44(0.34,0.58)$ & $<0.00001$ & $<0.00001$ & $87 \%$ & $\mathrm{R}$ \\
\hline & His/His vs. Arg/His+Arg/Arg & 19 & $0.68(0.61,0.77)$ & $<0.00001$ & $<0.00001$ & $71 \%$ & $\mathrm{R}$ \\
\hline \multirow[t]{5}{*}{ Non-Asian } & His vs. Arg & 2 & $0.93(0.59,1.47)$ & 0.75 & 0.46 & $0 \%$ & $\mathrm{~F}$ \\
\hline & His/His vs. Arg/Arg & 1 & $0.82(0.15,4.53)$ & 0.82 & NA & NA & \\
\hline & Arg/His vs. Arg/Arg & 2 & $0.95(0.57,1.56)$ & 0.83 & 0.46 & $0 \%$ & $\mathrm{~F}$ \\
\hline & $\mathrm{His} / \mathrm{His}+\mathrm{Arg} / \mathrm{His}$ vs. Arg/Arg & 3 & $0.59(0.23,1.48)$ & 0.26 & 0.02 & $75 \%$ & $\mathrm{R}$ \\
\hline & His/His vs. Arg/His+Arg/Arg & 1 & $0.84(0.15,4.64)$ & 0.84 & NA & NA & \\
\hline \multirow[t]{5}{*}{ China } & His vs. Arg & 11 & $0.64(0.56,0.73)$ & $<0.00001$ & $<0.00001$ & $78 \%$ & $\mathrm{R}$ \\
\hline & His/His vs. Arg/Arg & 11 & $0.37(0.29,0.50)$ & $<0.00001$ & $<0.00001$ & $79 \%$ & $\mathrm{R}$ \\
\hline & Arg/His vs. Arg/Arg & 11 & $0.47(0.35,0.63)$ & $<0.00001$ & $<0.00001$ & $77 \%$ & $\mathrm{R}$ \\
\hline & $\mathrm{His} / \mathrm{His}+\mathrm{Arg} / \mathrm{His}$ vs. Arg/Arg & 11 & $0.41(0.31,0.55)$ & $<0.00001$ & $<0.00001$ & $80 \%$ & $\mathrm{R}$ \\
\hline & His/His vs. Arg/His+Arg/Arg & 12 & $0.71(0.66,0.76)$ & $<0.00001$ & 0.16 & $29 \%$ & $\mathrm{~F}$ \\
\hline \multirow[t]{5}{*}{ Japan } & His vs. Arg & 4 & $0.55(0.51,0.60)$ & $<0.00001$ & 0.41 & $0 \%$ & $\mathrm{~F}$ \\
\hline & His/His vs. Arg/Arg & 4 & $0.26(0.18,0.38)$ & $<0.00001$ & 0.03 & $66 \%$ & $\mathrm{R}$ \\
\hline & Arg/His vs. Arg/Arg & 4 & $0.35(0.21,0.59)$ & $<0.00001$ & 0.0008 & $82 \%$ & $\mathrm{R}$ \\
\hline & $\mathrm{His} / \mathrm{His}+\mathrm{Arg} / \mathrm{His}$ vs. Arg/Arg & 5 & $0.31(0.22,0.44)$ & $<0.00001$ & 0.004 & $74 \%$ & $\mathrm{R}$ \\
\hline & His/His vs. Arg/His+Arg/Arg & 4 & $0.62(0.56,0.69)$ & $<0.00001$ & 0.65 & $0 \%$ & $\mathrm{~F}$ \\
\hline
\end{tabular}

NA not applicable, OR odds ratio, $95 \% \mathrm{Cl} 95 \%$ confidence interval, $P h$ and $l^{2}$ test of heterogeneity, $F$ fixed-effect model, $R$ random-effect model

\begin{tabular}{|c|c|c|c|c|c|c|c|c|c|}
\hline \multirow[b]{2}{*}{ Study or Subgroup } & \multicolumn{2}{|c|}{ Cases } & \multicolumn{2}{|c|}{ Controls } & \multicolumn{2}{|r|}{ Odds Ratio } & \multirow{2}{*}{\multicolumn{3}{|c|}{$\begin{array}{l}\text { Odds Ratio } \\
\mathrm{M}-\mathrm{H}, \text { Random, } 95 \% \mathrm{Cl}\end{array}$}} \\
\hline & Events & Total & Events & Total & Weight & M-H. Random. $95 \%$ Cl Year & & & \\
\hline Boonyaphiphat $\mathrm{P}$ & 116 & 404 & 195 & 522 & $5.1 \%$ & $0.68[0.51,0.89] 2002$ & $\mp$ & & \\
\hline Chao YC & 97 & 176 & 153 & 210 & $3.9 \%$ & $0.46[0.30,0.70] 2003$ & - & & \\
\hline Yokoyama T & 291 & 466 & 946 & 1220 & $5.6 \%$ & $0.48[0.38,0.61] 2003$ & - & & \\
\hline Chen HG & 168 & 274 & 197 & 274 & $4.4 \%$ & $0.62[0.43,0.89] 2005$ & - & & \\
\hline Yang $\mathrm{CX}$ & 233 & 330 & 776 & 988 & $5.1 \%$ & $0.66[0.50,0.87] 2005$ & - & & \\
\hline Yang SJ & 236 & 382 & 276 & 396 & $5.0 \%$ & $0.70[0.52,0.95] 2007$ & $\mp$ & & \\
\hline Guo YM & 101 & 160 & 744 & 960 & $4.5 \%$ & $0.50[0.35,0.71] 2008$ & - & & \\
\hline Lee $\mathrm{CH}$ & 429 & 812 & 945 & 1312 & $5.9 \%$ & $0.44[0.36,0.52] 2008$ & $=$ & & \\
\hline Akbai & 1212 & 1486 & 2125 & 2742 & $6.1 \%$ & $1.28[1.10,1.51] 2009$ & & * & \\
\hline Cui $R$ & 1383 & 2134 & 4238 & 5526 & $6.5 \%$ & $0.56[0.50,0.62] 2009$ & . & & \\
\hline Ding JH & 308 & 442 & 291 & 382 & $4.9 \%$ & $0.72[0.53,0.98] 2010$ & 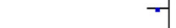 & & \\
\hline Tanaka F & 949 & 1484 & 1255 & 1640 & $6.2 \%$ & $0.54[0.47,0.64] 2010$ & $=$ & & \\
\hline lqbal B & 40 & 100 & 39 & 100 & $2.9 \%$ & $1.04[0.59,1.84] 2011$ & - & & \\
\hline Wang YL & 100 & 164 & 223 & 324 & $4.2 \%$ & $0.71[0.48,1.05] 2011$ & -1 & & \\
\hline Gu HY & 484 & 758 & 534 & 756 & $5.7 \%$ & $0.73[0.59,0.91] 2012$ & - & & \\
\hline Dura $\mathrm{P}$ & 20 & 692 & 23 & 858 & $2.7 \%$ & $1.08[0.59,1.98] 2013$ & - & & \\
\hline Gao Y & 2785 & 4196 & 3219 & 4526 & $6.5 \%$ & $0.80[0.73,0.88] 2013$ & - & & \\
\hline Wu M & 1019 & 1604 & 1430 & 2042 & $6.3 \%$ & $0.75[0.65,0.86] 2013$ & $=$ & & \\
\hline YeB & 1154 & 2002 & 1904 & 2782 & $6.4 \%$ & $0.63[0.56,0.71] 2014$ & $=$ & & \\
\hline Babiker $\mathrm{H}$ & 12 & 250 & 26 & 420 & $2.2 \%$ & $0.76[0.38,1.54] 2015$ & $\cdot$ & & \\
\hline Total $(95 \% \mathrm{Cl})$ & & 18316 & & 27980 & $100.0 \%$ & $0.67[0.59,0.76]$ & 4 & & \\
\hline Total events & 11137 & & 19539 & & & & & & \\
\hline $\begin{array}{l}\text { Heterogeneity: } \text { Tau }^{2}= \\
\text { Test for overall effect: }\end{array}$ & $\begin{array}{l}.06 ; \mathrm{Chi}^{2} \\
\mathrm{z}=6.17(\mathrm{P}\end{array}$ & $\begin{array}{l}=141.1 \\
0<0.00\end{array}$ & $\begin{array}{l}9, \mathrm{df}=19 \\
001)\end{array}$ & $9(P<0$. & .00001); I & & $\begin{array}{ccc}0.01 & 0.1 & 1 \\
\text { Favours [Cases] }\end{array}$ & $\begin{array}{c}10 \\
\text { Favours [con }\end{array}$ & $\begin{array}{r}100 \\
\text { trols] }\end{array}$ \\
\hline
\end{tabular}

Fig. 2 Meta-analysis of ADH1B Arg47His polymorphism and esophageal cancer risk in a random-effect model under the allelic models (His vs. Arg) 


\begin{tabular}{|c|c|c|c|c|c|c|c|c|}
\hline Study or Subgroup & $\begin{array}{l}\text { Cases } \\
\text { Events }\end{array}$ & Total & \multicolumn{2}{|c|}{ Controls } & Weight & $\begin{array}{l}\text { Odds Ratio } \\
\text { M-H, Random, } 95 \% \mathrm{Cl} \text { Year }\end{array}$ & \multicolumn{2}{|c|}{$\begin{array}{l}\text { Odds Ratio } \\
\text { M-H, Random, } 95 \% \mathrm{Cl}\end{array}$} \\
\hline \multicolumn{9}{|l|}{ 3.1.1 Asians } \\
\hline Yokoyama A & 56 & 112 & 381 & 526 & $5.1 \%$ & $0.38[0.25,0.58] 2001$ & - & \\
\hline Boonyaphiphat $\mathrm{P}$ & 101 & 202 & 167 & 261 & $5.3 \%$ & $0.56[0.39,0.82] 2002$ & - & \\
\hline Chao YC & 69 & 88 & 98 & 105 & $3.3 \%$ & $0.26[0.10,0.65] 2003$ & & \\
\hline Yokoyama T & 182 & 233 & 579 & 610 & $4.9 \%$ & $0.19[0.12,0.31] 2003$ & & \\
\hline Chen HG & 111 & 137 & 123 & 137 & $4.1 \%$ & $0.49[0.24,0.98] 2005$ & & \\
\hline Yang CX & 159 & 165 & 472 & 494 & $3.3 \%$ & $1.24[0.49,3.10] 2005$ & & \\
\hline Yang SJ & 158 & 191 & 176 & 198 & $4.5 \%$ & $0.60[0.33,1.07] 2007$ & & \\
\hline Guo YM & 63 & 80 & 456 & 480 & $4.2 \%$ & $0.20[0.10,0.38] 2008$ & 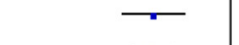 & \\
\hline Lee $\mathrm{CH}$ & 289 & 406 & 610 & 656 & $5.3 \%$ & $0.19[0.13,0.27] 2008$ & 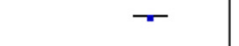 & \\
\hline Akbai & 722 & 743 & 1298 & 1371 & $4.9 \%$ & $1.93[1.18,3.17] 2009$ & & — \\
\hline Cui R & 873 & 1067 & 2612 & 2763 & $5.7 \%$ & $0.26[0.21,0.33] 2009$ & $=$ & \\
\hline Ding JH & 202 & 221 & 183 & 191 & $3.6 \%$ & $0.46[0.20,1.09] 2010$ & & \\
\hline Tanaka F & 593 & 742 & 771 & 820 & $5.4 \%$ & $0.25[0.18,0.36] 2010$ & - & \\
\hline lqbal B & 38 & 50 & 38 & 50 & $3.3 \%$ & $1.00[0.40,2.50] 2011$ & & \\
\hline Wang YL & 67 & 82 & 145 & 162 & $3.9 \%$ & $0.52[0.25,1.11] 2011$ & & \\
\hline Gu HY & 326 & 379 & 352 & 378 & $4.9 \%$ & $0.45[0.28,0.74] 2012$ & - & \\
\hline Gao $Y$ & 1846 & 2098 & 2064 & 2263 & $5.8 \%$ & $0.71[0.58,0.86] 2013$ & $\mp$ & \\
\hline Wu M & 664 & 802 & 920 & 1021 & $5.6 \%$ & $0.53[0.40,0.70] 2013$ & $\sigma$ & \\
\hline Ye B & 777 & 1001 & 1241 & 1391 & $5.7 \%$ & $0.42[0.33,0.53] 2014$ & $\bar{T}$ & \\
\hline Subtotal $(95 \% \mathrm{Cl})$ & & 8799 & & 13877 & $88.8 \%$ & $0.44[0.34,0.58]$ & & \\
\hline Total events & 7296 & & 12686 & & & & & \\
\hline \multicolumn{9}{|c|}{ Heterogeneity: $\mathrm{Tau}^{2}=0.27 ; \mathrm{Chi}^{2}=140.56, \mathrm{df}=18(\mathrm{P}<0.00001) ;\left.\right|^{2}=87 \%$} \\
\hline \multicolumn{9}{|c|}{ Test for overall effect: $Z=6.07(P<0.00001)$} \\
\hline \multicolumn{9}{|l|}{ 3.1.2 non-Asians } \\
\hline Hashibe M & 4 & 167 & 95 & 887 & $3.0 \%$ & $0.20[0.07,0.56] 2006$ & & \\
\hline Dura $P$ & 20 & 346 & 23 & 429 & $4.4 \%$ & $1.08[0.58,2.01] 2013$ & & \\
\hline Babiker $\mathrm{H}$ & 10 & 125 & 22 & 210 & $3.8 \%$ & $0.74[0.34,1.63] 2015$ & & \\
\hline Subtotal $(95 \% \mathrm{Cl})$ & & 638 & & 1526 & $11.2 \%$ & $0.59[0.23,1.48]$ & & \\
\hline Total events & 34 & & 140 & & & & & \\
\hline \multirow{2}{*}{\multicolumn{9}{|c|}{$\begin{array}{l}\text { Heterogeneity: } \mathrm{Tau}^{2}=0.50 ; \mathrm{Chi}^{2}=8.15, \mathrm{df}=2(P=0.02) ; I^{2}=75 \% \\
\text { Test for overall effect: } Z=1.13(P=0.26)\end{array}$}} \\
\hline & & & & & & & & \\
\hline Total $(95 \% \mathrm{Cl})$ & & 9437 & & 15403 & $100.0 \%$ & $0.46[0.36,0.59]$ & $\checkmark$ & \\
\hline Total events & 7330 & & 12826 & & & & & \\
\hline \multicolumn{7}{|c|}{ Heterogeneity: $\mathrm{Tau}^{2}=0.27 ; \mathrm{Chi}^{2}=152.82, \mathrm{df}=21(\mathrm{P}<0.00001) ; \mathrm{I}^{2}=86 \%$} & 0.010 .1 & 110 \\
\hline \multicolumn{7}{|c|}{ Test for overall effect: $Z=6.09(P<0.00001)$} & $\begin{array}{l}0.01 \\
\text { Favours [Cases] }\end{array}$ & Favours [controls] \\
\hline \multicolumn{9}{|c|}{ Test for subaroun differences: $\mathrm{Chi}^{2}=0.34 . \mathrm{df}=1(\mathrm{P}=0.56) . \mathrm{I}^{2}=0 \%$} \\
\hline $\begin{array}{l}\text { Fig. } 3 \text { Subgroup analysis } \\
\text { His vs. Arg/Arg) }\end{array}$ & ethnicity c & of $\mathrm{ADH}$ & 1B Arg47Hi & tis polym & horphism an & d esophageal cancer susceptibi & under the dor & nodel (His/His+Arg/ \\
\hline
\end{tabular}

which is in turn oxidized to aldehyde by aldehyde dehydrogenases (ALD) [59]. ADH1B and ALDH2 are the major enzymes involved in the alcohol-metabolizing pathways in humans. ADH1B is one member of $\mathrm{ADH}$ family and is expressed in the liver and presented in gastrointestinal tract. This gene is mainly contributed for ethanol to carcinogenic metabolite conversion during the elimination phage [60]. In addition, ADH1B expression could result in high blood acetaldehyde levels, which can easily give rise to DNA damage, and finally cause the cancer occurrence [61]. Genetic polymorphisms of ADH1B could modulate exposure levels to ethanol. ADH1B can include three alleles $\left(\mathrm{ADH} 2 * 1, \mathrm{ADH}_{2} * 2\right.$, and $\left.\mathrm{ADH} 2 * 3\right)$. Among which, the Arg47His variant (ADH2*2) was the most studied. The activity of ADH1B was increased by 40 -fold in
ADH1B His/His individuals [62], resulting in the fast accumulation of acetaldehyde.

SNPs of ADH1B gene (the major alcohol-metabolizing enzyme gene) may modify the effects of alcohol on metabolic and clinical phenotypes [63]. Blood ethanol concentrations of $\mathrm{ADH} 1 \mathrm{~B} * 2 / 22$ group were shown higher than those of ADH1B*1/*2 group [64]. ADH1B allele was associated with a reduction in alcohol consumption [65] and might be an important protective factor for alcoholic liver cirrhosis, especially for Asians [66]. ADH2*2 predicted less drinking and was a protective effect against heavy drinking in Jewish samples [67]. Heavy alcohol consumption and heavy smoking were shown to be associated with the increased risk of esophageal cancer particularly in individuals with the flushing response [68]. 


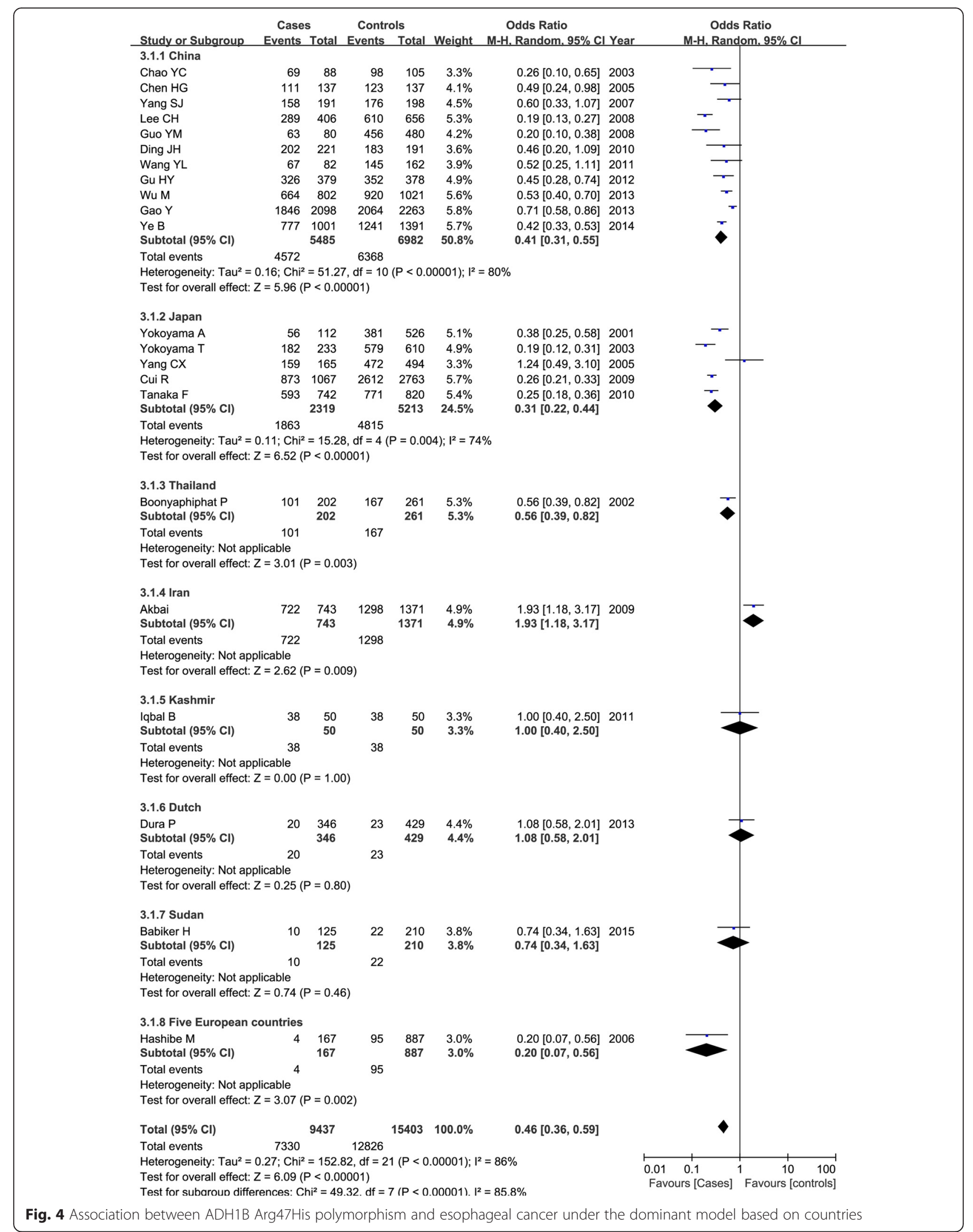




\begin{tabular}{|c|c|c|c|c|c|c|c|c|}
\hline Study or Subaroun & $\begin{array}{c}\text { Cases } \\
\text { Events T }\end{array}$ & Total E & Control & & Weiaht $N$ & $\begin{array}{l}\text { Odds Ratio } \\
\text { M-H. Random, } 95 \% \text { Cl Year }\end{array}$ & $\begin{array}{r}\text { Odds } \\
\text { M-H.Rand }\end{array}$ & Ratio \\
\hline Boonyaphiphat $\mathrm{P}$ & 18 & 53 & 48 & 133 & $11.2 \%$ & $0.91[0.47,1.78] 2002$ & & 1ini. 3010 \\
\hline Yokoyama $\mathrm{T}$ & 1 & 5 & 4 & 142 & $1.7 \%$ & $8.63[0.78,95.67] 2003$ & & \\
\hline Chao YC & 2 & 29 & 7 & 105 & $3.4 \%$ & $1.04[0.20,5.28] 2003$ & & \\
\hline Chen HG & 0 & 13 & 4 & 41 & $1.2 \%$ & $0.31[0.02,6.12] \quad 2005$ & & \\
\hline Yang $\mathrm{SJ}$ & 37 & 80 & 53 & 128 & $13.0 \%$ & $1.22[0.69,2.14] \quad 2007$ & & $=$ \\
\hline Guo YM & 1 & 14 & 12 & 285 & $2.2 \%$ & $1.75[0.21,14.50] 2008$ & & \\
\hline Lee $\mathrm{CH}$ & 8 & 63 & 27 & 471 & $8.9 \%$ & $2.39[1.04,5.53] 2008$ & & - \\
\hline Cui R & 62 & 406 & 109 & 2031 & $17.2 \%$ & $3.18[2.28,4.43] 2009$ & & $=$ \\
\hline Ding JH & 4 & 96 & 3 & 94 & $3.8 \%$ & $1.32[0.29,6.06] 2010$ & & \\
\hline Gu HY & 25 & 252 & 17 & 269 & $11.6 \%$ & $1.63[0.86,3.10] 2012$ & & $=$ \\
\hline WuM & 42 & 330 & 54 & 591 & $15.4 \%$ & $1.45[0.95,2.22] 2013$ & & $=$ \\
\hline Hou AM & 30 & 61 & 20 & 67 & $10.4 \%$ & $2.27[1.10,4.70] 2014$ & & $\cdot$ \\
\hline Total $(95 \% \mathrm{Cl})$ & & 1402 & & $4357 \quad 1$ & $100.0 \%$ & $1.71[1.23,2.38]$ & & 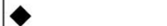 \\
\hline Total events & 230 & & 358 & & & & & \\
\hline $\begin{array}{l}\text { Heterogeneity: } \mathrm{Tau}^{2}=C \\
\text { Test for overall effect: } z\end{array}$ & $\begin{array}{l}14 ; \mathrm{Chi}^{2}= \\
=3.19(\mathrm{P}\end{array}$ & $\begin{array}{l}=22.48, \\
=0.001\end{array}$ & $\begin{array}{l}\mathrm{df}=11(\mathrm{~d} \\
1)\end{array}$ & $(P=0.02$ & $.02) ; 1^{2}=51 \%$ & & $\begin{array}{lc} & 1 \\
0.01 & 0.1 \\
\text { Favours [Cases] }\end{array}$ & \begin{tabular}{|ccc}
1 & 10 & 100 \\
1 & \\
Favours [controls]
\end{tabular} \\
\hline Study or Suboroup & $\begin{array}{c}\text { Cases } \\
\text { Events }\end{array}$ & Total & $\begin{array}{l}\text { Contr } \\
\text { Events }\end{array}$ & rols & al Weight & $\begin{array}{l}\text { Odds Ratio } \\
\text { M-H. Fixed. } 95 \% \text { Cl Year }\end{array}$ & $\begin{array}{r}\text { Odds } \\
\text { M-H. Fixe }\end{array}$ & $\begin{array}{l}\text { s Ratio } \\
\text { ed. } 95 \% \mathrm{Cl}\end{array}$ \\
\hline Boonyaphiphat P & 83 & 149 & 46 & 128 & $\begin{array}{ll}8 & 14.1 \% \\
\end{array}$ & $2.24[1.38,3.64] 2002$ & & $\rightarrow$ \\
\hline Yokoyama T & 50 & 216 & 27 & 477 & $7 \quad 8.3 \%$ & $5.02[3.04,8.28] 2003$ & & - \\
\hline Chao YC & 17 & 59 & 12 & 87 & $4.4 \%$ & $2.53[1.10,5.80] 2003$ & & - \\
\hline Chen HG & 17 & 70 & 4 & 43 & $2.4 \%$ & $3.13[0.98,10.03] 2005$ & & \\
\hline Yang SJ & 30 & 111 & 11 & 70 & $6.3 \%$ & $1.99[0.92,4.28] \quad 2007$ & & - \\
\hline Guo YM & 16 & 66 & 12 & 195 & $2.9 \%$ & $4.88[2.17,10.98] 2008$ & & - \\
\hline Lee $\mathrm{CH}$ & 109 & 343 & 19 & 185 & $5 \quad 10.8 \%$ & $4.07[2.40,6.89] 2008$ & & $=$ \\
\hline Cui R & 92 & 410 & 42 & 730 & $\begin{array}{ll}0 & 15.0 \%\end{array}$ & $4.74[3.21,6.99] 2009$ & & $=$ \\
\hline Ding JH & 15 & 125 & 5 & 97 & $3.2 \%$ & $2.51[0.88,7.17] 2010$ & & \\
\hline Gu HY & 28 & 127 & 9 & 109 & $4.8 \%$ & $3.14[1.41,7.00] 2012$ & & $\cdots$ \\
\hline Wu M & 87 & 436 & 46 & 415 & $\begin{array}{ll}5 & 24.2 \% \\
\end{array}$ & $2.00[1.36,2.94] 2013$ & & $\mp$ \\
\hline Hou AM & 21 & 52 & 8 & 40 & $0 \quad 3.5 \%$ & $2.71[1.05,7.02] 2014$ & & \\
\hline Total $(95 \% \mathrm{Cl})$ & & 2164 & & 2576 & $6 \quad 100.0 \%$ & $3.15[2.66,3.74]$ & & - \\
\hline Total events & 565 & & 241 & & & & & \\
\hline $\begin{array}{l}\text { Heterogeneity: } \mathrm{Chi}^{2}= \\
\text { Test for overall effect: }\end{array}$ & $\begin{array}{l}8.75, \mathrm{df}= \\
z=13.17\end{array}$ & $\begin{array}{l}=11(P=) \\
(P<0.0\end{array}$ & $\begin{array}{l}=0.07) ; 1 \\
00001)\end{array}$ & $I^{2}=41 \%$ & & & $\begin{array}{ll} & 1 \\
0.01 & 0.1 \\
\text { Favours [Cases] }\end{array}$ & $\begin{array}{ccc} & 1 & 10 \\
1 & 100 \\
\text { Favours [Controls] }\end{array}$ \\
\hline
\end{tabular}

Fig. 5 Forest plot of the combined effect of ADH1B Arg47His polymorphism and alcohol drinking condition and esophageal cancer risk under the Arg/Arg versus Arg/His+His/His model (a non-drinkers; $\mathbf{b}$ drinkers)

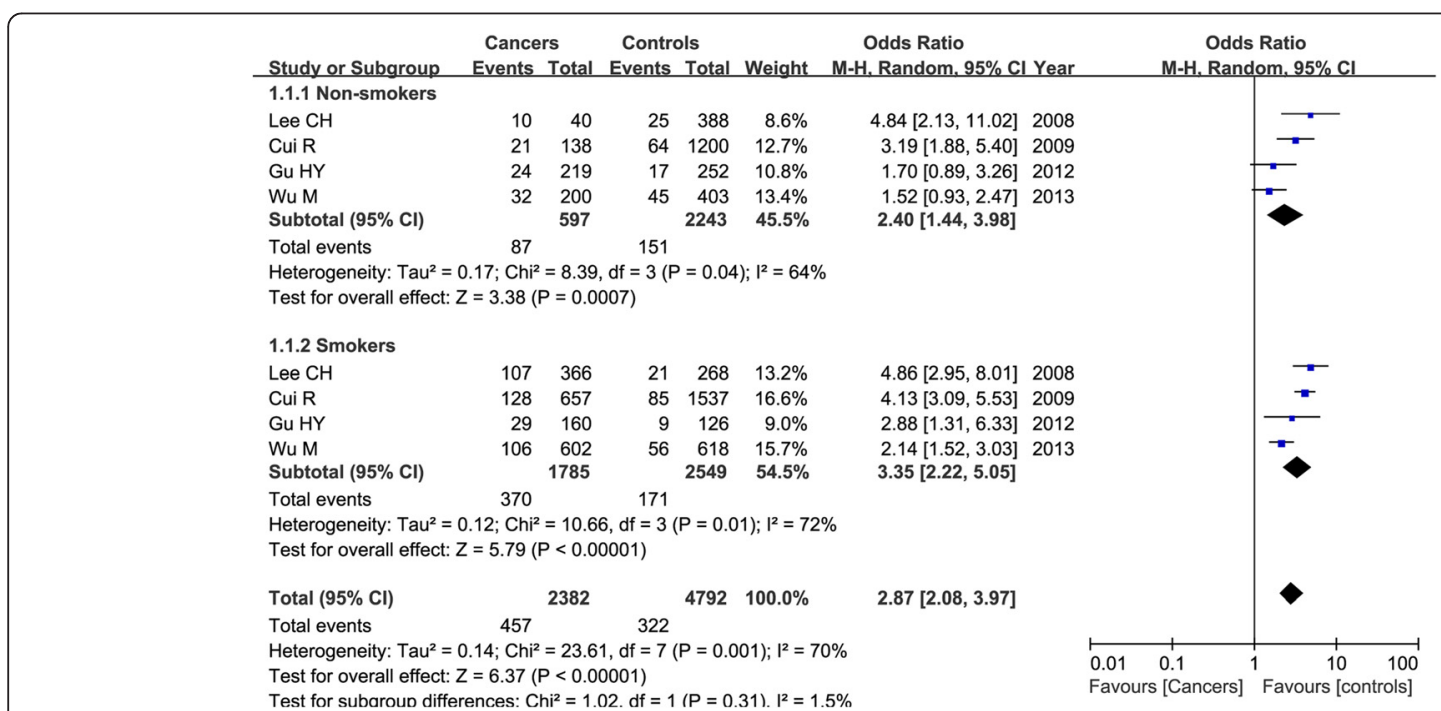

Fig. 6 Meta-analysis of the combined effect of ADH1B Arg47His polymorphism and smoking status and esophageal cancer risk under the Arg/Arg versus Arg/His+His/His model 


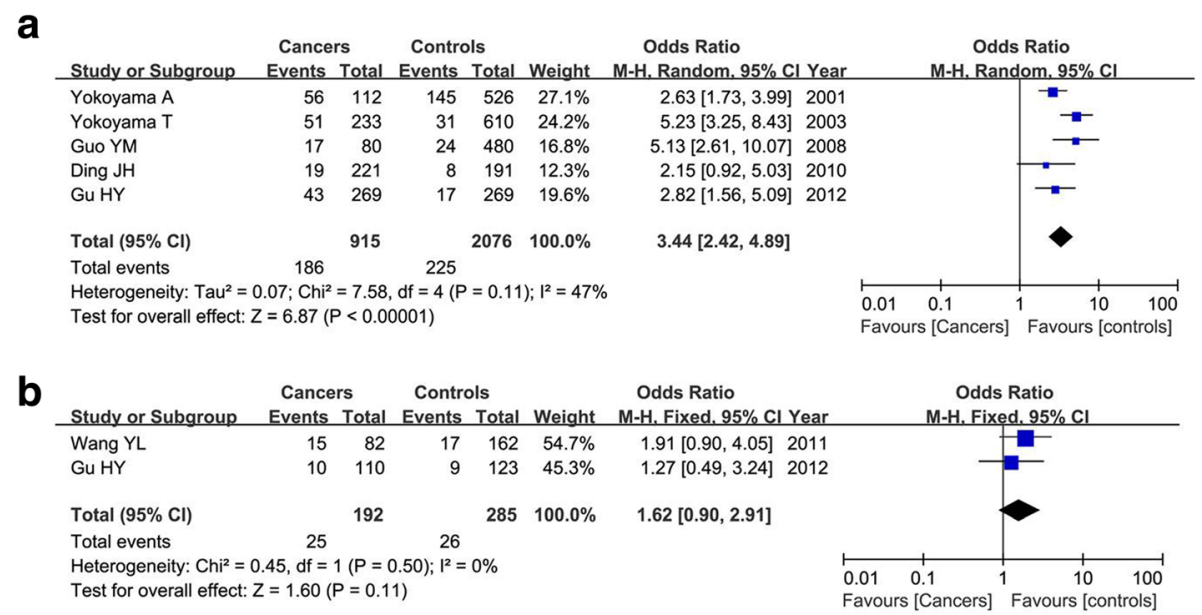

Fig. 7 Forest plot of the combined effect of ADH1B Arg47His polymorphism and gender variance and esophageal cancer risk under the Arg/Arg versus $\mathrm{Arg} / \mathrm{His}+\mathrm{His} / \mathrm{His}$ model (a males; $\mathbf{b}$ females)

Previous studies have found that ADH1B Arg47His polymorphism was associated with the pathogenesis of cancers such as colorectal cancer [69] and head and neck cancer [70], while no positive correlation was found in hepatocellular carcinoma [71] and gastric cancer [72]. Several studies have identified the association between ADH1B polymorphism and esophagus cancer susceptibility. Chen et al. have observed that individuals with ADH1BArg/Arg genotype had a 3.99-fold risk of developing esophageal cancer compared with those with ADH1B His/His genotype [73]. Li et al. have demonstrated that $\mathrm{ADH} 2 * 1$ was associated with the increased risk of oesophageal cancer, possibly due to the tolerance of the carriers of these alleles to alcohol consumption compared to those with high-activity alleles $\mathrm{ADH} 2 * 2$ which are associated with higher production of the unpleasant acetaldehyde intermediate [74]. Oze et al. found that the magnitude of effect of ADH1B polymorphisms was greater in subjects who were heavy drinkers, heavy smokers, and had esophageal cancer [75]. Consumption of tobacco and alcohol, coupled with ADH1B genotypes, determines a substantial magnitude of tumorigenetic effect on earlier age ESCC diagnosis [76].

Several limitations were presented in this metaanalysis. Firstly, most of the included studies were conducted in the Asian population, while other ethnicities should be considered in the future. Secondly, the stages of patients with esophageal cancer could not be extracted from the included studies, which might limit the application of our results. Thirdly, the combined effect of ADH1B Arg47His variant and age group also should be focused on. Lastly, other polymorphisms in ADH1B

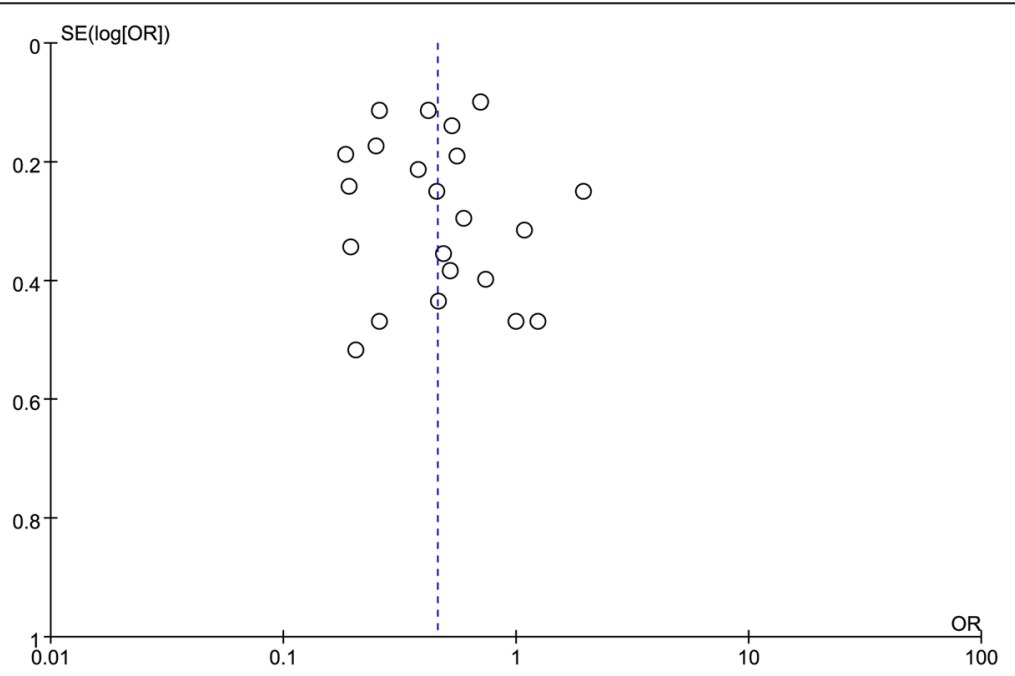

Fig. 8 Funnel plot of the publication bias in this meta-analysis under the dominant model in total population (His/His+Arg/His vs. Arg/Arg) 
gene or other $\mathrm{ADH}$ genes which might alter the metabolism of alcohol should be included.

\section{Conclusions}

In conclusion, our results suggested that ADH1B Arg47His polymorphism might be a protective factor on esophageal cancer susceptibility in Asians. The Arg/Arg genotypes combined with alcohol drinking, tobacco smoking, and males might be strongly increased the risk of esophageal cancer. However, further studies with more ethnicities should be taken into account in future researches.

\section{Abbreviations}

AC, adenocarcinomas; $A D H$, alcohol dehydrogenase gene; ADH1B, alcohol dehydrogenase 1B; ALD, aldehyde dehydrogenases; $\mathrm{Cl}$, confidence interval: OR, odds ratio; SCC, squamous cell carcinomas; SNPs, single nucleotide polymorphisms

\section{Acknowledgements}

None.

\section{Funding}

No funding supported this study.

\section{Availability of data and materials}

The data will not be shared because not all authors agreed with this.

\section{Authors' contributions}

NM, SYN, and TX designed and conducted the experiments. $\mathrm{BH}$ and $\mathrm{CL}$ surveyed the literature and developed the text of the manuscript. XYS helped in the statistical analysis. NM, BH, and TX refined the write-up. All authors read and approved the final manuscript.

\section{Competing interests}

The authors declare that they have no competing interests.

\section{Consent for publication}

Not applicable.

\section{Ethics approval and consent to participate}

Not applicable.

\section{Author details}

'Department of Cardiothoracic Surgery, Yongchuan Hospital of Chongqing Medical University, No. 439 Xuanhua Road, Yongchuan District, Chongqing 402160, China. ${ }^{2}$ Department of Infectious Disease, Yongchuan Hospital of Chongqing Medical University, Chongqing 402160, China.

Received: 6 May 2016 Accepted: 8 July 2016

Published online: 22 July 2016

\section{References}

1. Schweigert M, Dubecz A, Stein HJ. Oesophageal cancer-an overview. Nat Rev Gastroenterol Hepatol. 2013:10:230-44.

2. Napier KJ, Scheerer M, Misra S. Esophageal cancer: a review of epidemiology, pathogenesis, staging workup and treatment modalities. World J Gastrointest Oncol. 2014;6:112.

3. Stewart BW, Wild CP. World cancer report 2014. Geneva: International Agency for Research on Cancer; 2014.

4. Kornblum N. Esophageal Cancer[M], Geriatric Gastroenterology. New York: Springer; 2012. p. 571-579.

5. Arnal MJD, Arenas ÁF, Arbeloa ÁL. Esophageal cancer: risk factors, screening and endoscopic treatment in Western and Eastern countries. World J Gastroenterol. 2015;21:7933

6. Gao Y-B, Chen Z-L, Li J-G, Hu X-D, Shi X-J, Sun Z-M, Zhang F, Zhao Z-R, Li Z-T, Liu Z-Y. Genetic landscape of esophageal squamous cell carcinoma. Nat Genet. 2014.
7. Tang W, Chen Z, Lin K, Su M, Au W. Development of esophageal cancer in Chaoshan region, China: association with environmental, genetic and cultural factors. Int J Hyg Environ Health. 2015;218:12-8.

8. Whiteman DC, Sadeghi S, Pandeya N, Smithers BM, Gotley DC, Bain CJ, Webb PM, Green AC. Combined effects of obesity, acid reflux and smoking on the risk of adenocarcinomas of the oesophagus. Gut. 2008;57:173-80.

9. Ferlay J, Soerjomataram I, Dikshit R, Eser S, Mathers C, Rebelo M, Parkin DM, Forman D, Bray F. Cancer incidence and mortality worldwide: sources, methods and major patterns in GLOBOCAN 2012. Int J Cancer. 2015;136:E359-86.

10. Giri S, Pathak R, Aryal MR, Karmacharya P, Bhatt VR, Martin MG. Incidence trend of esophageal squamous cell carcinoma: an analysis of Surveillance Epidemiology, and End Results (SEER) database. Cancer Causes Control. 2015;26:159-61.

11. Andrici J, Eslick GD. Epidemiology and Risk Factors for Esophageal Cancer[M], Esophageal Cancer. Heidelberg: Springer International Publishing; 2015. p. 1-23.

12. Siegel RL, Miller KD, Jemal A. Cancer statistics, 2015. CA Cancer J Clin. 2015;65:5-29.

13. Merkow RP, Bilimoria KY, Keswani RN, Chung J, Sherman KL, Knab LM, Posner MC, Bentrem DJ. Treatment trends, risk of lymph node metastasis, and outcomes for localized esophageal cancer. J Natl Cancer Inst. 2014;106:dju133.

14. Lao-Sirieix P. Fitzgerald RC. Screening for oesophageal cancer. Nat Rev Clin Oncol. 2012:9:278-87.

15. Kaz AM, Grady WM. Epigenetic biomarkers in esophageal cancer. Cancer Lett. 2014:342:193-9.

16. Pennathur A, Gibson MK, Jobe BA, Luketich JD. Oesophageal carcinoma. Lancet. 2013:381:400-12.

17. Whiteman DC. Esophageal cancer: priorities for prevention. Curr Epidemiol Rep. 2014:1:138-48.

18. Chattopadhyay I. A brief overview of genetics of esophageal squamous cell carcinoma. J Cell Sci Molecul Biol. 2014;1:103.

19. Zuo L, Wang K, Zhang X-Y, Pan X, Wang G, Tan Y, Zhong C, Krystal JH, Zhang $\mathrm{H}$, Luo X. Association between common alcohol dehydrogenase gene (ADH) variants and schizophrenia and autism. Hum Genet. 2013;132:735-43.

20. Luo X, Kranzler HR, Zuo L, Zhang H, Wang S, Gelernter J. ADH7 variation modulates extraversion and conscientiousness in substance-dependent subjects. Am J Med Genet B Neuropsychiatr Genet. 2008;147:179-86.

21. Svensson S, Some M, Lundsjö A, Helander A, Cronholm T, Höög JO. Activities of human alcohol dehydrogenases in the metabolic pathways of ethanol and serotonin. Eur J Biochem. 1999;262:324-9.

22. Strommer J. The plant ADH gene family. Plant J. 2011;66:128-42.

23. Yoshida A. Genetics of human alcohol-metabolizing enzymes. Prog Nucleic Acid Res Mol Biol. 1991;40:225-67.

24. Bosron WF, Li TK. Genetic polymorphism of human liver alcohol and aldehyde dehydrogenases, and their relationship to alcohol metabolism and alcoholism. Hepatology. 1986;6:502-10.

25. Bierut LJ, Goate AM, Breslau N, Johnson EO, Bertelsen S, Fox L, Agrawal A, Bucholz KK, Grucza R, Hesselbrock V. ADH1B is associated with alcohol dependence and alcohol consumption in populations of European and African ancestry. Mol Psychiatry. 2012;17:445-50.

26. Li D, Zhao H, Gelernter J. Strong association of the alcohol dehydrogenase $1 \mathrm{~B}$ gene (ADH1B) with alcohol dependence and alcohol-induced medical diseases. Biol Psychiatry. 2011;70:504-12.

27. Ito $\mathrm{H}$, Oze I, Hosono S, Watanabe M, Tanaka H, Matsuo K. The risk prediction for esophageal cancer by drinking, smoking, and the polymorphisms of ALDH2 and ADH1B. Cancer Res. 2015:75:4618.

28. Ma W-J, Lv G-D, Zheng S-T, Huang C-G, Liu Q, Wang X, Lin R-Y, Sheyhidin I, Lu $X-M$. DNA polymorphism and risk of esophageal squamous cell carcinoma in a population of North Xinjiang, China. World J Gastroenterol. 2010;16:641.

29. Zhang L, Jiang Y, Wu Q, Li Q, Chen D, Xu L, Zhang C, Zhang M, Ye L. Geneenvironment interactions on the risk of esophageal cancer among Asian populations with the G48A polymorphism in the alcohol dehydrogenase-2 gene: a meta-analysis. Tumor Biol. 2014;35:4705-17.

30. Zhang G, Mai R, Huang B. ADH1B Arg47His polymorphism is associated with esophageal cancer risk in high-incidence Asian population: evidence from a meta-analysis. PLoS One. 2010;5

31. Yang S-J, Yokoyama A, Yokoyama T, Huang Y-C, Wu S-Y, Shao Y, Niu J, Wang J Liu Y, Zhou X-Q. Relationship between genetic polymorphisms of ALDH2 and ADH1B and esophageal cancer risk: a meta-analysis. World J Gastroenterol. 2010;16:4210. 
32. Zhang $\mathrm{H}-\mathrm{Z}$, Jin $\mathrm{G}-\mathrm{F}$, Shen H-B. Epidemiologic differences in esophageal cancer between Asian and Western populations. Chin J Cancer. 2012;31:281.

33. Hou A, Tong X, Miao Z. Correlation of alcohol dehydrogenase and aldehyde dehydrogenase polymorphisms, and alcohol drinking in esophageal cancer. China J Pharm Econ. 2014;223-224.

34. Chen H. A match case-control study on esophageal cancer susceptibility with lifestyle habits and genetic polymorphisms of $\mathrm{ALDH} 2$ and $\mathrm{ADH} 2$. Sichuan University; Master's degree; 2005.

35. Cui R, Kamatani Y, Takahashi A, Usami M, Hosono N, Kawaguchi T, Tsunoda T, Kamatani N, Kubo M, Nakamura Y. Functional variants in ADH1B and ALDH2 coupled with alcohol and smoking synergistically enhance esophageal cancer risk. Gastroenterology. 2009;137:1768-75.

36. Lee CH, Lee JM, Wu DC, Goan YG, Chou SH, Wu I, Kao EL, Chan TF, Huang MC, Chen PS. Carcinogenetic impact of ADH1B and ALDH2 genes on squamous cell carcinoma risk of the esophagus with regard to the consumption of alcohol, tobacco and betel quid. Int J Cancer. 2008;122:1347-56.

37. Tanaka F, Yamamoto K, Suzuki S, Inoue H, Tsurumaru M, Kajiyama Y, Kato $H$, Igaki H, Furuta K, Fujita $H$. Strong interaction between the effects of alcohol consumption and smoking on oesophageal squamous cell carcinoma among individuals with $\mathrm{ADH} 1 \mathrm{~B}$ and/or $\mathrm{ALDH} 2$ risk alleles. Gut. 2010:59:1457-64.

38. Hashibe M, Boffetta P, Zaridze D, Shangina O, Szeszenia-Dabrowska N, Mates D, Janout V, Fabiánová E, Bencko V, Moullan N. Evidence for an important role of alcohol-and aldehyde-metabolizing genes in cancers of the upper aerodigestive tract. Cancer Epidemiol Biomarkers Prev. 2006;15:696-703.

39. Wu M, Chang SC, Kampman E, Yang J, Wang XS, Gu XP, Han RQ, Liu AM, Wallar G, Zhou JY. Single nucleotide polymorphisms of ADH1B, ADH1C and ALDH2 genes and esophageal cancer: A population-based case-control study in China. Int J Cancer. 2013;132:1868-77

40. Wang Y, Ji R, Wei X, Gu L, Chen L, Rong Y, Wang R, Zhang Z, Liu B, Xia S. Esophageal squamous cell carcinoma and $\mathrm{ALDH} 2$ and $\mathrm{ADH} 1 \mathrm{~B}$ polymorphisms in Chinese females. Asian Pac J Cancer Prev. 2011;12:2065-8.

41. Guo Y-M, Wang Q, Liu Y-Z, Chen H-M, Qi Z, Guo Q-H. Genetic polymorphisms in cytochrome P4502E1, alcohol and aldehyde dehydrogenases and the risk of esophageal squamous cell carcinoma in Gansu Chinese males. World J Gastroenterol. 2008;14:1444.

42. Gu H, Gong D, Ding G, Zhang W, Liu C, Jiang P, Chen S, Chen Y. A variant allele of $\mathrm{ADH} 1 \mathrm{~B}$ and $\mathrm{ALDH} 2$, is associated with the risk of esophageal cancer. Exp Ther Med. 2012:4:135-40.

43. Akbari MR, Malekzadeh $R$, Shakeri $R$, Nasrollahzadeh D, Foumani M, Sun $Y$, Pourshams A, Sadjadi A, Jafari E, Sotoudeh M. Candidate gene association study of esophageal squamous cell carcinoma in a high-risk region in Iran. Cancer Res. 2009;69:7994-8000.

44. Babiker H. Genetic polymorphisms in alcohol and xenobiotic metabolizing enzymes as risk factors of oesophageal cancer in Sudan. 2015. UOFK.

45. Ye B, Ji C-Y, Zhao Y, Li W, Feng J, Zhang X. Single nucleotide polymorphism at alcohol dehydrogenase-1B is associated with risk of esophageal squamous cell carcinoma. Cancer Cell Int. 2014;14:1-7.

46. Gao Y, He Y, Xu J, Xu L, Du J, Zhu C, Gu H, Ma H, Hu Z, Jin G. Genetic variants at $4 q 21,4 q 23$ and $12 q 24$ are associated with esophageal squamous cell carcinoma risk in a Chinese population. Hum Genet. 2013;132:649-56.

47. Dura P, Berkers T, van Veen EM, Salomon J, te Morsche RH, Roelofs HM, Kristinsson JO, Wobbes T, Witteman BJ, Tan AC. Polymorphisms in alcoholmetabolizing enzymes and esophageal carcinoma susceptibility: a Dutch Caucasian case-control study. J Hum Genet. 2013;58:742-8.

48. lqbal B, Dar NAG. Comparison of alcohol dehydrogenase 2 and aldehyde dehydrogenase 2 polymorphism in esophageal cancer cases vs controls in Kashmir. 2011

49. J-h D, S-p L, H-x C, Wu J-Z, Gao C-m, Y-t L, J-n Z, Chang J, Yao G-h. Alcohol dehydrogenase-2 and aldehyde dehydrogenase-2 genotypes, alcohol drinking and the risk for esophageal cancer in a Chinese population. J Hum Genet. 2010;55:97-102.

50. Yang S-J, Wang H-Y, Li X-Q, Du H-Z, Zheng C-J, Chen H-G, Mu X-Y, Yang C-X. Genetic polymorphisms of $\mathrm{ADH} 2$ and $\mathrm{ALDH} 2$ association with esophageal cancer risk in southwest China. World J Gastroenterol. 2007;13:5760-4.

51. Yang C, Matsuo K, Ito H, Hirose K, Wakai K, Saito T, Shinoda M, Hatooka S, Mizutani K, Tajima K. Esophageal cancer risk by ALDH2 and $\mathrm{ADH} 2$ polymorphisms and alcohol consumption: exploration of gene-environment and gene-gene interactions. Asian Pac J Cancer Prev. 2005;6:256.

52. Chao Y-C, Wang L-S, Hsieh T-Y, Chu C-W, Chang F-Y, Chu H-C. Chinese alcoholic patients with esophageal cancer are genetically different from alcoholics with acute pancreatitis and liver cirrhosis. Am J Gastroenterol. 2000;95:2958-64.

53. Boonyaphiphat $P$, Thongsuksai $P$, Sriplung $H$, Puttawibul $P$. Lifestyle habits and genetic susceptibility and the risk of esophageal cancer in the Thai population. Cancer Lett. 2002;186:193-9.

54. Yokoyama T, Yokoyama A, Kato H, Tsujinaka T, Muto M, Omori T, Haneda T, Kumagai Y, Igaki H, Yokoyama M. Alcohol flushing, alcohol and aldehyde dehydrogenase genotypes, and risk for esophageal squamous cell carcinoma in Japanese men. Cancer Epidemiol Biomarkers Prev. 2003;12:1227-33.

55. Yokoyama A, Muramatsu T, Omori T, Yokoyama T, Matsushita S, Higuchi S, Maruyama K, Ishii H. Alcohol and aldehyde dehydrogenase gene polymorphisms and oropharyngolaryngeal, esophageal and stomach cancers in Japanese alcoholics. Carcinogenesis. 2001;22:433-9.

56. Zhang Y. Epidemiology of esophageal cancer. World J Gastroenterol. 2013;19:5598.

57. Cheung WY, Liu G. Genetic variations in esophageal cancer risk and prognosis. Gastroenterol Clin North Am. 2009;38:75-91.

58. Jelski W, Orywal K, Laniewska M, Szmitkowski M. The diagnostic value of alcohol dehydrogenase (ADH) isoenzymes and aldehyde dehydrogenase $(\mathrm{ALDH})$ measurement in the sera of gastric cancer patients. Clin Exp Med. 2010;10:215-9.

59. De Smidt O, Du Preez JC, Albertyn J. The alcohol dehydrogenases of Saccharomyces cerevisiae: a comprehensive review. FEMS Yeast Res. 2008:8:967-78.

60. Lao-Sirieix P, Caldas C, Fitzgerald RC. Genetic predisposition to gastro-oesophageal cancer. Curr Opin Genet Dev. 2010;20:210-7.

61. Singh NP, Khan A. Acetaldehyde: genotoxicity and cytotoxicity in human lymphocytes. Mut Res DNA Repair. 1995;337:9-17.

62. Lee S-L, Höög J-O, Yin S-J. Functionality of allelic variations in human alcohol dehydrogenase gene family: assessment of a functional window for protection against alcoholism. Pharmacogenet Genomics. 2004;14:725-32.

63. Kiage JN, James LO, Kabagambe EK. Genetic modification of the effects of alcohol on metabolic and clinical phenotypes: a review. Curr Nutr Rep. 2014;3:213-22.

64. Kang G, Bae KY, Kim SW, Kim J, Shin HY, Kim JM, Shin IS, Yoon JS, Kim JK. Effect of the allelic variant of alcohol dehydrogenase ADH1B* 2 on ethanol metabolism. Alcohol Clin Exp Res. 2014;38:1502-9.

65. Ferrari P, McKay J, Jenab M, Brennan P, Canzian F, Vogel U, Tjønneland A, Overvad K, Tolstrup JS, Boutron-Ruault M. Alcohol dehydrogenase and aldehyde dehydrogenase gene polymorphisms, alcohol intake and the risk of colorectal cancer in the European Prospective Investigation into Cancer and Nutrition study. Eur J Clin Nutr. 2012;66:1303-8.

66. He L, Deng T, Luo H-S. Genetic polymorphism in alcohol dehydrogenase 2 (ADH2) gene and alcoholic liver cirrhosis risk. Int J Clin Exp. 2015;8:7786.

67. Hasin D, Aharonovich E, Liu X, Mamman Z, Matseoane K, Carr L, Li T-K. Alcohol and ADH2 in Israel: Ashkenazis, Sephardics, and recent Russian immigrants. Am J Psychiatry. 2014.

68. Ishiguro S, Sasazuki S, Inoue M, Kurahashi N, Iwasaki M, Tsugane S, Group JS. Effect of alcohol consumption, cigarette smoking and flushing response on esophageal cancer risk: a population-based cohort study (JPHC study). Cancer Lett. 2009;275:240-6.

69. Crous-Bou M, Rennert G, Cuadras D, Salazar R, Cordero D, Rennert HS, Lejbkowicz F, Kopelovich L, Lipkin SM, Gruber SB. Polymorphisms in alcohol metabolism genes $\mathrm{ADH} 1 \mathrm{~B}$ and $\mathrm{ALDH} 2$, alcohol consumption and colorectal cancer. 2013.

70. Ji YB, Tae K, Ahn TH, Lee SH, Kim KR, Park CW, Park BL, Shin HD. ADH1B and ALDH2 polymorphisms and their associations with increased risk of squamous cell carcinoma of the head and neck in the Korean population. Oral Oncol. 2011;47:583-7.

71. Liu S, Cui Y, Yang B, Chai P, Su Z, Zhang Q, Zheng D, Li R, Yu G. Relationship between $\mathrm{ADH} 2 \mathrm{Arg} 47 \mathrm{His}$ variation and hepatocellular carcinoma susceptibility: a meta analysis. Int J Clin Exp. 2015:8:9543.

72. Wang H-L, Zhou P-Y, Liu P, Zhang Y. ALDH2 and ADH1 genetic polymorphisms may contribute to the risk of gastric cancer: a meta-analysis. PLoS One. 2014;9.

73. Chen YJ, Chen C, Wu DC, Lee CH, Wu Cl, Lee JM, Goan YG, Huang SP, Lin CC, Li TC. Interactive effects of lifetime alcohol consumption and alcohol and aldehyde dehydrogenase polymorphisms on esophageal cancer risks. Int J Cancer. 2006;119:2827-31.

74. Li D-P, Dandara C, Walther G, Parker MI. Genetic polymorphisms of alcohol metabolising enzymes: their role in susceptibility to oesophageal cancer. Clin Chem Lab Med. 2008;46:323-8. 
75. Oze I, Matsuo K, Suzuki T, Kawase T, Watanabe M, Hiraki A, Ito H, Hosono S, Ozawa T, Hatooka S. Impact of multiple alcohol dehydrogenase gene polymorphisms on risk of upper aerodigestive tract cancers in a Japanese population. Cancer Epidemiol Biomarkers Prev. 2009;18:3097-102.

76. Lee CH, Wu DC, Wu I, Goan YG, Lee JM, Chou SH, Chan TF, Huang HL, Hung YH, Huang MC. Genetic modulation of ADH1B and ALDH2 polymorphisms with regard to alcohol and tobacco consumption for younger aged esophageal squamous cell carcinoma diagnosis. Int J Cancer. 2009;125:1134-42.

Submit your next manuscript to BioMed Central and we will help you at every step:

- We accept pre-submission inquiries

- Our selector tool helps you to find the most relevant journal

- We provide round the clock customer support

- Convenient online submission

- Thorough peer review

- Inclusion in PubMed and all major indexing services

- Maximum visibility for your research

Submit your manuscript at www.biomedcentral.com/submit 